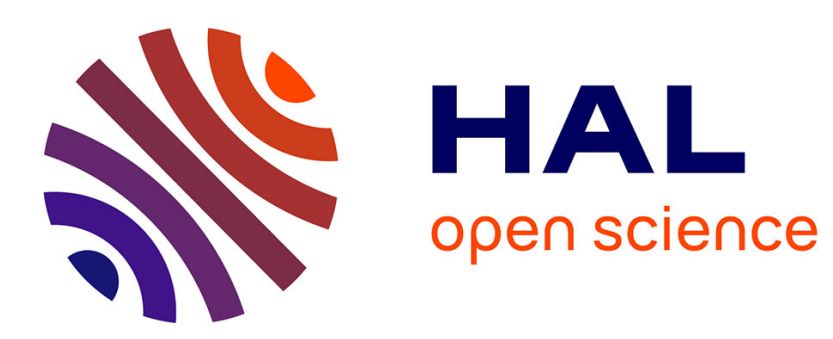

\title{
Les potentialités de production du blé d'hiver en moyenne montagne du Massif Central
}

\author{
Eugène Triboi, Louis Gachon, Jean Morizet
}

\section{To cite this version:}

Eugène Triboi, Louis Gachon, Jean Morizet. Les potentialités de production du blé d'hiver en moyenne montagne du Massif Central. Agronomie, 1985, 5 (2), pp.135-142. hal-00884742

\section{HAL Id: hal-00884742 \\ https://hal.science/hal-00884742}

Submitted on 1 Jan 1985

HAL is a multi-disciplinary open access archive for the deposit and dissemination of scientific research documents, whether they are published or not. The documents may come from teaching and research institutions in France or abroad, or from public or private research centers.
L'archive ouverte pluridisciplinaire HAL, est destinée au dépôt et à la diffusion de documents scientifiques de niveau recherche, publiés ou non, émanant des établissements d'enseignement et de recherche français ou étrangers, des laboratoires publics ou privés. 


\section{Les potentialités de production du blé d'hiver en moyenne montagne du Massif Central}

Eugène TRIBOI, Louis GACHON \& Jean MORIZET

I.N.R.A., Station d'Agronomie, F 63039 Clermont-Ferrand Cedex

RÉSUMÉ Les potentialités de production du blé d'hiver (cv. "Clément ») sont étudiées à l'altitude de $900 \mathrm{~m}$ dans un
dispositif lysimétrique où les facteurs de variation sont les suivants : le système de culture, l'épaisseur du sol,
la fumure azotée (minérale ou organique) et l'irrigation.
Elles approchent $100 \mathrm{q}$ de grain (à $15 \mathrm{p}$. $100 \mathrm{~d}$ 'eau) à l'ha dès lors que sont assurés les besoins en eau et en
azote. Pour la variété « Clément " ces derniers sont de l'ordre de $3,0 \mathrm{~kg}$ de $\mathrm{N}$ par quintal de grain à $15 \mathrm{p}$. 100
d'humidité. La forte pluviosité hivernale ( 400 à $600 \mathrm{~mm}$ ) provoque un important lessivage d'octobre à avril et
des risques élevés de pertes d'azote minéral. La meilleure valorisation de la fumure azotée par le blé d'hiver
implique le fractionnement de cette fumure.

Mots clés additionnels : Système de culture, épaisseur du sol, eau, lysimétrie, fertilisation azotée.

The yield potential of winter wheat (cv. 'Clement') was studied in lysimetric conditions at an altitude of $900 \mathrm{~m}$, where the factors of variation were cropping system, soil thickness, nitrogen fertilizer (mineral and organic), irrigation. When water and nitrogen were not limiting factors the yield potential was about 10 tonne/ha $(85$ p. 100 D.M.). For cv. 'Clement' the nitrogen need was $30 \mathrm{~kg}$ of $\mathrm{N}$ per tonne of grain. Leaching of mineral nitrogen was possible from October to April when the rainfall is high (400 to $600 \mathrm{~mm})$. In these conditions, split applications give the best utilization of nitrogen fertilizer.

Additional key words : Cropping system, soil thickness, water, lysimeter, nitrogen fertilizer.

\section{INTRODUCTION}

Dans le Massif Central, on admet généralement que la moyenne montagne débute vers $600 \mathrm{~m}$, soit approximativement en Auvergne avec la limite des sédiments tertiaires et de leurs terrains primaires encaissants, et que son altitude supérieure correspond à la possibilité du seigle de parvenir à maturité, c'està-dire vers $1100 \mathrm{~m}$ au nord et $1300 \mathrm{~m}$ au sud.

Le Massif Central possède actuellement les $2 / 3$ des surfaces montagnardes françaises consacrées à la céréaliculture. Deux exploitations sur trois cultivent encore des céréales. Mais les surfaces diminuent progressivement en raison des faibles rendements moyens (20-30 q/ha) (LAFARGE, 1983a).

Cependant, des rendements élevés sont réalisables. Ainsi, les expérimentations et enquêtes en exploitation effectuées ces dernières années soit par LAFARGE $(1983 b)$, soit par l'Institut Technique des Céréales et des Fourrages (ITCF) ou les firmes d'engrais, révèlent des rendements maxima dépassant parfois $60 \mathrm{q} / \mathrm{ha}$, exceptionnellement 70 q. Selon LAFARGE, l'alimentation azotée et les mauvaises herbes sont les principaux facteurs de limitation des rendements et la céréaliculture a sa place en montagne en raison de la souplesse qu'elle confère à l'adaptation du système de culture aux conditions individuelles d'exploitation.

Le présent mémoire rapporte les résultats d'une culture de blé d'hiver en 1981-1982 sur le dispositif lysimétrique permanent de Theix, situé à l'altitude de $900 \mathrm{~m}$ sur le plateau cristallin des Monts Dômes, soit à $15 \mathrm{~km}$ au Sud-Ouest de Clermont-Ferrand.

Ce dispositif lourd a été mis en place en 1968 dans le but d'étudier :

- le bilan biogéochimique des principaux éléments de la biosphère ;

- les potentalités des cultures et des prairies en relation avec l'épaisseur du sol ;

- le bilan hydrique des diverses productions. 


\section{MATÉRIEL ET MÉTHODES}

\section{A. Le dispositif expérimental}

Dans son ensemble, le dispositif comprend 50 cuves étanches en polyester stratifié, laquées intérieurement et extérieurement, de section carrée égale à $3 \mathrm{~m}^{2}$, de profondeurs différentes, à fond en dièdre légèrement incliné suivant une diagonale jusqu'à un orifice d'évacuation des eaux recueillies à l'aval par gravité.

Dans ces cuves ont été reconstitués divers profils de la manière suivante :

- au fond, un lit de $10 \mathrm{~cm}$ d'épaisseur moyenne de gravillons quartzeux destiné à faciliter l'écoulement des eaux infiltrées ;

- au-dessus, une épaisseur variable de «terre de sous-sol » prélevée sur place ;

- puis, $40 \mathrm{~cm}$ au maximum de «terre de surface » prélevée également sur la parcelle.

Dans la partie en prairie permanente, des plaques de gazon naturel prélevées à proximité immédiate ont assuré la couverture de surface $(0-10 \mathrm{~cm})$.

Enfin, le bord supérieur des cuves dépasse de 3 à $6 \mathrm{~cm}$ la surface du sol naturel pour éviter toute circulation hydrique superficielle entre l'intérieur et l'extérieur des cases.

Pour limiter les influences microclimatiques réciproques (effets d'oasis) consécutives aux croissances différentes induites par les traitements appliqués, chaque cuve est enterrée au centre d'un carré de $16 \mathrm{~m}^{2}$, zone qui bénéficie des mêmes techniques culturales et qui constitue ainsi une zone de protection.

Les paramètres testés dans ce dispositif sont les suivants :

\section{Le système de culture}

- la prairie permanente (système P.P.) ;

- une succession culturale comprenant une prairie temporaire de 4 ans rompue par 2 années de cultures annuelles : système prairie assolée (P.A.) ;

- une rotation de 6 ans à dominante de cultures fourragères annuelles (système C.A:) incluant simultanément les cultures du système précéclent.

\section{L'épaisseur du sol}

Au sein de chaque système cultural, on a fait varier l'épaisseur du sol selon une échelle adaptée à la puissance du système racinaire des cultures pratiquées, soit :

- sous P.P. : 20,40 et $80 \mathrm{~cm}$,

- sous P.A. : 40,80 et $120 \mathrm{~cm}$,

- sous C.A. : $40,80,120$ et $160 \mathrm{~cm}$.

Les cultures pratiquées sur chacun de ces systèmes reçoivent une fumure azotée standard, minérale et organique (NL).

\section{La fumure azotée met en jeu}

- 2 formes : minérale $(\mathrm{N})$ (exclusivement ammonitrate 33 p. 100 ), organique (L) (engrais de ferme sous forme de lisier ou de fumier)

et -2 niveaux : absence (T), présence

agissant seuls ou en combinaison à l'intérieur de 4 traitements : T-L-N-LN.

Les fumures appliquées sont évidemment variables selon les cultures. En principe, elles ont pour ambition de satisfaire les besoins de productions intensives dans le contexte pédoclimatique local. Au cours des années, elles ont subi des ajustements successifs pour tenir compte des résultats observés.

Pour ne pas alourdir exagérément le dispositif, cette interaction $\mathrm{N}$ minéral $\times \mathrm{N}$ organique est étudiée exclusivement sur le sol de $80 \mathrm{~cm}$ d'épaisseur commun aux 3 systèmes culturaux.

\section{L'irrigation}

Un traitement supplémentaire (80 LNI), mis en œuvre sur l'épaisseur de $80 \mathrm{~cm}$, permet de mesurer les effets de l'irrigation appliquée de manière à satisfaire la demande climatique, déterminée à partir de la consommation en eau mesurée sur des évapotranspiromètres intégrés au dispositif, portant par conséquent les mêmes cultures.

Enfin, il convient de signaler que tous ces traitements bénéficient de fumures phosphopotassiques largement calculées et comprennent 2 répétitions.

\section{B. Les conditions climatiques et pédologiques}

\section{Le climat}

Les conditions climatiques moyennes issues des données recueillies sur le site de 1970 à 1982, ainsi que celles de septembre 1981 à août 1982 sont précisées dans le tableau 1.

TABLEAU 1

Conditions climatiques sur le site expérimental (1970-1982 et 1981-82).

Climatic conditions of experimental field (1970-1982 and 1981-82).

\begin{tabular}{|c|c|c|c|c|c|c|c|c|c|c|c|c|c|c|}
\hline Mois & & $S$ & $\mathrm{O}$ & $\mathrm{N}$ & $\mathrm{D}$ & $\mathbf{J}$ & $\mathrm{F}$ & M & A & $\mathbf{M}$ & $J$ & $\mathrm{~J}$ & A & Année \\
\hline \multirow{2}{*}{ Pluie (mm) } & moy. & 80,3 & 88,6 & 56,4 & 65,7 & 59,7 & 50,8 & 56,1 & 45,9 & 94,2 & 74,7 & 62,3 & 80,4 & 815,1 \\
\hline & $81-82$ & 207,7 & 52,0 & 21,6 & 150,3 & 122,5 & 18,0 & 69,3 & 13,3 & 41,5 & 87,5 & 52,0 & 64,7 & 900,3 \\
\hline $\begin{array}{l}\text { Température } \\
\text { moyenne }\left({ }^{\circ} \mathrm{C}\right)\end{array}$ & moy. & 11,9 & 7,8 & 4,0 & 1,7 & 0,9 & 1,6 & 2,7 & 5,0 & 9,3 & 12,6 & 14,7 & 14,8 & 7,2 \\
\hline sous abri & $81-82$ & 11,8 & 7,5 & 5,0 & 1,6 & 4,4 & 3,8 & 2,4 & 5,7 & 10,5 & 14,7 & 18,0 & 14,9 & 8,3 \\
\hline
\end{tabular}


Le climat est donc relativement froid avec des températures moyennes de l'ordre de $15{ }^{\circ} \mathrm{C}$ seulement en juillet-août et très faiblement positives pendant les 3 mois d'hiver. Malgré l'altitude, il est modérément humide car le site, placé à l'Est des appareils volcaniques de la chaîne des Puys, bénéficie d'un phénomène d'abri vis-à-vis de l'onde pluviale se déplaçant d'Ouest en Est sous l'effet des vents dominants.

Par rapport à ces conditions moyennes, l'année culturale 1981-82 est marquée par :

- une très forte irrégularité de la pluie : largement excédentaire en septembre, décembre et janvier en alternance avec des épisodes anormalement secs en novembre, février, avril et mai. Ce régime pluvial engendre la saturation quasi permanente de la capacité au champ, du début septembre à la mi-avril (date d'arrêt définitif du lessivage) et des drainages globaux compris entre 515 et $569 \mathrm{~mm}$ selon l'épaisseur du sol ;

- des températures relativement douces en hiver et au printemps, toujours supérieures à la moyenne, de janvier à la récolte, à l'exception du mois de mars, conditions qui ont assuré le maintien des peuplements initiaux.

\section{Le sol}

Il s'agit d'un sol brun acide, modérément lessivé, représentatif du type pédologique le plus fréquent en moyenne montagne du centre de la France. Il est ici formé à partir de l'arène d'altération des granites porphyroïdes riches en plagioclases du socle cristallin des Monts-Dômes. Ses caractéristiques physicochimiques déterminées lors de la mise en place du dispositif sont indiquées au tableau 2 .

Sa texture associe une charge sableuse dominante à un complexe d'altération normalement développé, peu désaturé et à base de kaolinite. Ce sol présente sur toute son épaisseur des teneurs satisfaisantes en matières organiques et en phosphore qui témoignent à la fois de son bon entretien chimique passé, lié à la proximité des bâtiments d'exploitation (restitutions d'engrais de ferme), et des phénomènes de colluvionnement ayant contribué à sa genèse malgré la pente modérée de la parcelle (6 p. 100, face au Nord).

\section{L'expérimentation 1981-82 sur blé d'hiver}

\section{Conduite de la culture}

La variété fourragère "Clément " a été semée le 15 octobre 1981 dans les 2 systèmes de culture (C.A. et P.A.), à la même densité de 400 grains $/ \mathrm{m}^{2}$. La levée s'est effectuée le 2 novembre dans de bonnes conditions, aboutissant à un peuplement homogène de $341 \pm 11$ et $342 \pm 11$ pieds au $\mathrm{m}^{2}$, dans les systèmes C.A. et P.A. respectivement.

Dans ces 2 systèmes, l'orge de printemps constituait le même précédent cultural immédiat. Par contre, l'ante-précédent de 1980 était différent, soit en C.A., une vesce-avoine, soit en P.A., une prairie temporaire de 5 ans (fétuque élevée + trèfle blanc) retournée au printemps 1981 une dizaine de jours avant le semis de l'orge.

Les traitements avec l'azote ont reçu les fumures suivantes exprimées en $\mathrm{kg}$ de $\mathrm{N} / \mathrm{ha}$ :

- $170 \mathrm{~kg}$ provenant du fumier le 2 octobre 1981 ;

- $230 \mathrm{~kg} \mathrm{~N}$ de l'ammonitrate fractionnés en 3 apports : $30 \mathrm{~kg}$ le 4 novembre $1981,50 \mathrm{~kg}$ le 15 mars 1982 et $150 \mathrm{~kg}$ le 26 mai 1982, ce dernier au cours de la montaison.

Un désherbage chimique à l'Oxytril $\mathrm{M}$ a été effectué le 12 mai 1872 (début montaison) ainsi qu'un traitement antifongique à base de Bayleton le 9 juin (début épiaison). Enfin, le blé a été récolté le 18 août.

TABLEAU 2

Caractéristiques édaphiques (p. I 000 de terre fine sèche).

Edaphic characteristics (per 1000 parts of fine dry soil).

\begin{tabular}{|c|c|c|c|c|c|c|}
\hline \multirow{3}{*}{$\begin{array}{l}\text { Système cultural } \\
\text { Profondeur }(\mathrm{cm})\end{array}$} & \multirow{2}{*}{\multicolumn{2}{|c|}{$\begin{array}{c}\text { Prairie permanente } \\
\text { P.P. }\end{array}$}} & \multicolumn{4}{|c|}{ Prairie assolée et cultures annuelles } \\
\hline & & & & & & \\
\hline & $10-40$ & $40-80$ & $0-40$ & $40-80$ & $80-120$ & $120-160$ \\
\hline \multicolumn{7}{|l|}{ Analyse physique } \\
\hline Argile $\quad 0-2 \mu$ & 133 & 120 & 167 & 151 & 166 & 168 \\
\hline Limon & 130 & 142 & 162 & 194 & 191 & 178 \\
\hline \multirow{3}{*}{$\begin{array}{c}20-50 \mu \\
50-200 \mu \\
200-2000 \mu\end{array}$} & 90 & 79 & 86 & 95 & 91 & 78 \\
\hline & 126 & 109 & 128 & 138 & 131 & 115 \\
\hline & 500 & 539 & 405 & 380 & 375 & 398 \\
\hline Mat. Org. totales & 22,1 & 13,9 & 28,5 & 20,8 & 20,0 & 20,2 \\
\hline \multicolumn{7}{|l|}{ Analyse chimique } \\
\hline $\mathrm{C} / \mathrm{N}$ & 9,3 & 9,8 & 10,0 & 10,4 & 10,0 & 9,7 \\
\hline $\mathrm{pH}$ eau & 5,9 & 6,4 & 6,1 & 6,35 & 6,4 & 6,4 \\
\hline $\mathrm{P}_{2} \mathrm{O}_{5}$ (DYER) & 0,19 & 0,28 & 0,30 & 0,33 & 0,24 & 0,17 \\
\hline $\mathrm{K}_{2} \mathrm{O}$ échangeable & 0,27 & 0,24 & 0,39 & 0,41 & 0,42 & 0,39 \\
\hline C.E.C. (mev.) & 124 & 108 & 158 & 159 & 160 & 186 \\
\hline $\mathrm{Ca}(\mathrm{mev})$. & 47,5 & 59,0 & 66,6 & 66,0 & 66,5 & 66,5 \\
\hline Mg (mev.) & 14,1 & 16,6 & 16,4 & 19,3 & 19,0 & 18,1 \\
\hline $\mathrm{K}$ (mev.) & 5,7 & 4,3 & 8,0 & 8,8 & 9,0 & 8,25 \\
\hline $\mathrm{Na}(\mathrm{mev})$. & 0,7 & 0,7 & 0,9 & 1,2 & 0,9 & 0,9 \\
\hline Taux de saturation & 0,68 & 0,81 & 0,87 & 0,95 & 0,96 & 0,94 \\
\hline
\end{tabular}


TABLEAU 3

Rendements en matière sèche et exportations d'azote. Dry matter yield and nitrogen outputs.

\begin{tabular}{|c|c|c|c|c|c|c|c|c|c|c|}
\hline \multirow{2}{*}{$\begin{array}{l}\text { Epaisseur } \\
\text { sol (cm) }\end{array}$} & \multirow{2}{*}{ Traitement } & \multirow{2}{*}{$\begin{array}{l}\text { Système } \\
\text { cultural }\end{array}$} & \multicolumn{3}{|c|}{ Rendement ( $\mathrm{q} / \mathrm{ha}$ ) } & \multicolumn{2}{|c|}{$N(\%$ M.S. $)$} & \multicolumn{3}{|c|}{$\mathrm{N}$ dans la récolte $(\mathrm{kg} / \mathrm{ha})$} \\
\hline & & & grain & paille & total & grain & paille & grain & paille & total \\
\hline \multirow{2}{*}{40} & \multirow{2}{*}{ LN } & $\mathrm{CA}$ & 34,0 & 46,9 & 80,9 & 2,69 & 0,49 & 91,5 & 23,0 & 115 \\
\hline & & PA & 39,0 & 51,0 & 90,0 & 2,68 & 0,48 & 104,5 & 24,5 & 129 \\
\hline \multirow{10}{*}{80} & \multirow{2}{*}{$\mathrm{T}$} & $\mathrm{CA}$ & 15,9 & 20,5 & 36,4 & 1,76 & 0,38 & 28,0 & 7,8 & 36 \\
\hline & & PA & 26,9 & 34,7 & 61,6 & 1,70 & 0,31 & 45,7 & 10,8 & 57 \\
\hline & \multirow{2}{*}{$\mathrm{L}$} & $\mathrm{CA}$ & 25,9 & 34,4 & 60,3 & 1,52 & 0,25 & 39,4 & 8,6 & 48 \\
\hline & & $\mathrm{PA}$ & 38,8 & 50,0 & 88,8 & 1,64 & 0,28 & 63,6 & 14,0 & 78 \\
\hline & \multirow{2}{*}{$N$} & CA & 47,7 & 58,1 & 105,8 & 2,29 & 0,40 & 109,2 & 23,2 & 132 \\
\hline & & PA & 51,1 & 67,6 & 118,7 & 2,44 & 0,48 & 124,7 & 32,4 & 157 \\
\hline & \multirow{2}{*}{$\mathrm{LN}$} & $\mathrm{CA}$ & 51,2 & 68,3 & 119,5 & 2,43 & 0,50 & 124,4 & 34,2 & 159 \\
\hline & & PA & 54,6 & 76,7 & 131,3 & 2,55 & 0,45 & 139,2 & 34,5 & 174 \\
\hline & \multirow{2}{*}{ LNI } & $\mathrm{CA}$ & 77,1 & 94,8 & 171,9 & 2,18 & 0,50 & 168,1 & 47,4 & 216 \\
\hline & & PA & 79,8 & 108,2 & 188,0 & 2,30 & 0,53 & 183,5 & 57,3 & 241 \\
\hline \multirow{2}{*}{120} & \multirow{2}{*}{ LN } & $C A$ & 69,2 & 107,0 & 176,2 & 2,50 & 0,49 & 173,0 & 52,4 & 225 \\
\hline & & PA & 72,4 & 107,5 & 179,9 & 2,64 & 0,52 & 191,1 & 55,9 & 247 \\
\hline 160 & LN & $C A$ & 79,8 & 104,8 & 184,6 & 2,31 & 0,53 & 184,3 & 55,5 & 240 \\
\hline
\end{tabular}

\section{Contrôles effectués}

Les bilans hydriques et azotés ont été calculés à partir des mesures suivantes :

- volume et concentration des eaux de pluie et de lessivage (recueils hebdomadaires et analyses mensuelles sur échantillon moyen à partir de fractions aliquotes) ;

- consommation d'eau des cultures :

- soit directement sur les évapotranspiromètres,

- soit indirectement en prenant en compte la pluie (P), le lessivage (L), l'irrigation éventuelle (I) et la variation du stock hydrique $(\Delta S)$ du sol suivie par voie neutronique; d'où on obtient :

$$
\mathrm{ETR}=\mathrm{P}+\mathrm{I}+\Delta \mathrm{S}-\mathrm{L},
$$

- biomasse et composition des récoltes.

\section{RÉSULTATS ET DISCUSSION}

\section{A. Effets des traitements sur la production et l'absorption d'azote}

Sur l'ensemble du dispositif, la production et l'azote retrouvé dans les parties aériennes ont varié considérablement (tabl. 3). Les rendements exprimés en matière sèche sont passés de 15,9 à $79,8 \mathrm{q} /$ ha pour le grain et de 36,4 à $188,0 \mathrm{q} / \mathrm{ha}$ pour la biomasse aérienne totale. Dans celle-ci, l'azote' a fluctué entre 36 et $247 \mathrm{~kg} / \mathrm{ha}$.

Il est intéressant de comparer l'effet de la fumure minérale azotée à celui de la fumure organique au sein du sous-ensemble constitué par les sols de $80 \mathrm{~cm}$ d'épaisseur (tabl. 4).

Les $230 \mathrm{~kg}$ d'azote de la fumure minérale ont engendré un gain de 28,6 q de grain dans le système C.A. et de 20,0 q dans le système P.A. correspondant à l'absorption supplémentaire d'azote de 103,5 et $98 \mathrm{~kg}$ respectivement retrouvés dans l'ensemble de la récolte.

\section{TABLEAU 4}

Effets comparés de $N$ minéral et de $N$ organique selon le système de culture sur la production de grain et l'azote retrouvé dans la récolte.

Compared effects of mineral and organic nitrogen on grain yield and nitrogen recovery, according to cropping system.

\begin{tabular}{|c|c|c|c|c|}
\hline & \multicolumn{2}{|c|}{$\begin{array}{l}\text { Rendement du grain } \\
\text { (M.S. en } q / h a)\end{array}$} & \multicolumn{2}{|c|}{$\begin{array}{c}\mathrm{N} \text { dans la récolte } \\
(\mathrm{kg} / \mathrm{ha})\end{array}$} \\
\hline & C.A. & P.A. & C.A. & P.A. \\
\hline $\begin{array}{l}\text { Traitements } \quad \mathrm{T} \text { et } \mathrm{L} \\
\mathrm{N} \text { et } \mathrm{LN} \\
\text { Effet de } N \text { minéral }\end{array}$ & $\begin{array}{l}20,9 \\
49,5 \\
28,6\end{array}$ & $\begin{array}{l}32,9 \\
52,9 \\
20,0\end{array}$ & $\begin{array}{r}42,0 \\
145,5 \\
103,5\end{array}$ & $\begin{array}{r}67,5 \\
165,5 \\
98\end{array}$ \\
\hline $\begin{array}{c}\text { Traitements et } \mathrm{N} \\
\mathrm{L} \text { et } \mathrm{L} N \\
\text { Effet de } N \text { organique }\end{array}$ & $\begin{array}{c}31,8 \\
38,55 \\
6,8\end{array}$ & $\begin{array}{r}39,0 \\
46,7 \\
7,7\end{array}$ & $\begin{array}{r}84,0 \\
103,5 \\
19,5\end{array}$ & $\begin{array}{r}107,0 \\
126,0 \\
19,0\end{array}$ \\
\hline
\end{tabular}

Les $170 \mathrm{~kg}$ d'azote de la fumure organique ont exercé des effets beaucoup plus faibles, de l'ordre de 7 à $8 \mathrm{q}$ de grain, pour près de $20 \mathrm{~kg}$ supplémentaires de $\mathrm{N}$ absorbé. Pour le grain, on observe une nette interaction puisque le grain de rendement chute de 10-12 q sans $\mathrm{N}$ minéral à $3,5 \mathrm{q}$ seulement en présence de $\mathrm{N}$ minéral. Ces observations confirment à la fois le moindre coefficient d'utilisation de l'azote organique et l'interaction classique entre les 2 formes. Parmi les causes de cette moindre efficience, il faut souligner que l'apport de fumier effectué quelques jours avant le semis a provoqué des pertes supplémentaires d'environ $7,5 \mathrm{~kg}$ d'azote nitrique, peu variables selon les traitements, sous l'effet du lessivage hivernal.

En reprenant les résultats du tableau 3, on constate que l'épaisseur $d u$ sol a engendré les écarts les plus spectaculaires puisque le rendement du grain croît linéairement de 34,0 à $79,8 \mathrm{q}$ avec une pente moyenne de 0,39 ou $0,42 \mathrm{q}$ par $\mathrm{cm}$ supplémentaire de sol selon 


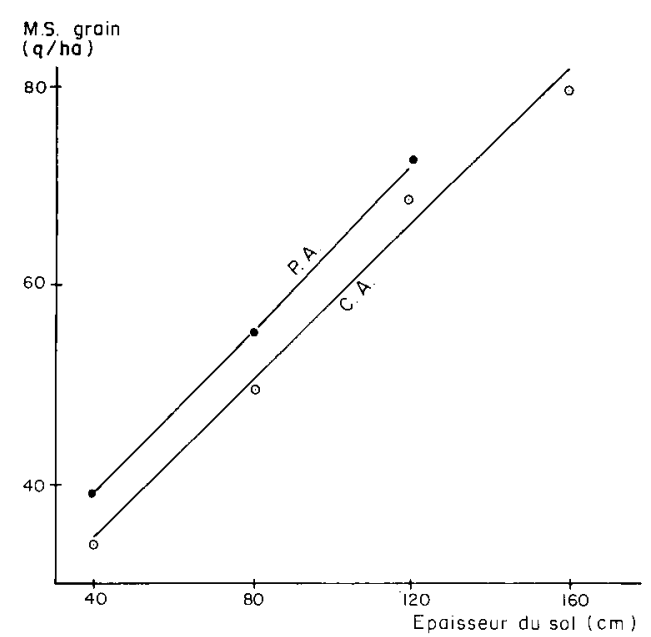

Figure 1

Relations entre le rendement du grain et l'épaisseur du sol selon le système de culture.

P.A. : prairie assolée; C.A. : cultures fourragères annuelles.

Relationships between grain yield and soil thickness according to cropping system.

le système de culture (fig. 1). On a :

en C.A. : MS grain $(\mathrm{q} / \mathrm{ha})=0,39$ Epaisseur $(\mathrm{cm})+19,7$ avec $n=4$ et $r=0,99$;

en P.A. : MS grain $(\mathrm{q} / \mathrm{ha})=0,42$ Epaisseur $(\mathrm{cm})+21,9$ avec $n=3$ et $r=1,00$.

L'influence de cette condition est à attribuer à la capacité globale de rétention en eau puisque l'irrigation accroît fortement à la fois la biomasse et le prélèvement d'azote.

On voit ainsi que les 2 facteurs, alimentation azotée et alimentation hydrique, ont induit une variation très importante des rendements de grain et de paille et de l'absorption d'azote.

A l'opposé, le système de culture a engendré un effet beaucoup plus limité au bénéfice de la prairie assolée (P.A.). Tous traitements confondus, cet effet se traduit par des écarts approximatifs de $6 \mathrm{q}$ de grain et de $22 \mathrm{~kg}$ d'azote prélevé, conséquence d'une plus forte fourniture d'azote minéral et, secondairement, d'une amélioration de la capacité de rétention pour l'eau, attestée par une tendance générale à la réduction du lessivage hydrique. On est surpris de constater une aussi faible arrière-action de la prairie temporaire retournée au cours du printemps 1981, notamment si on compare ces résultats à ceux de JACQUARD \& CROISIER (1970) obtenus, il est vrai, sous des conditions climatiques très différentes.

\section{B. Relation entre rendement et alimentation hydrique}

L'irrigation de $193 \mathrm{~mm}$ appliquée sur le seul traitement $80 \mathrm{LN}$ a augmenté le rendement du grain de $25 \mathrm{q}$ (moyenne des 2 systèmes de culture). Il en ressort une efficience de 0,130 par mm d'eau qui apparaît faible au premier abord.

A partir de la cessation du drainage en avril (peuplement en fin de tallage) et jusqu'à la récolte, les variations du profil hdyrique ont été suivies hebdomadairement sur 9 parcelles permettant d'établir le bilan hydrique et l'évapotranspiration réelle (ETR) au cours de cette phase terminale du cycle du blé. De l'analyse de ces bilans d'eau, il ressort que l'irrigation a augmenté l'ETR de $143 \mathrm{~mm}$ qui conduit à un coefficient apparent d'utilisation de l'eau de 74 p. 100. La différence d'eau non consommée s'explique par la variation du stock au sein du sol au cours de la période des contrôles et par quelques écarts dans les lessivages résiduels.

D'une manière générale, on constate qu'il existe une étroite relation linéaire entre le rendement du grain et l'ETR (fig. 2). On a : M.S. grain (q/ha) =0,19 ETR $(\mathrm{mm})-14,2$ avec $\mathrm{n}=9$ et $\mathrm{r}=0,98$.

L'importance relative du terme libre tient au fait que cette relation statistique ne considère que l'eau consommée postérieurement au 5 avril, début des contrôles. Il en résulte que l'efficience de l'eau apparaît d'autant meilleure que le rendement est plus élevé, en variant de 0,14 à 0,17 .

La biomasse totale est liée à l'ETR de manière tout à fait similaire :

M.S. totale aérienne $=0,46$ ETR $-33,6$

avec

$$
\mathrm{n}=9 \text { et } \mathrm{r}=0,96 \text {. }
$$

Conjointement, l'irrigation a accru l'exportation d'azote de 57 à $67 \mathrm{~kg}$ qu'il convient d'attribuer :

- soit à une plus intense minéralisation de $\mathrm{N}$ organique du sol, qu'elle qu'en soit son origine (humus, corps microbiens, résidus culturaux, engrais de ferme...) ;

- soit à une meilleure utilisation de l'offre du sol en azote minéral ( $\mathrm{N}$ posthivernal et $\mathrm{N}$ de la fumure) en considérant comme négligeable $\mathrm{N}$ minéral apporté par l'eau d'irrigation, de l'ordre de $5 \mathrm{~kg}$;

- soit à la combinaison de ces divers processus.

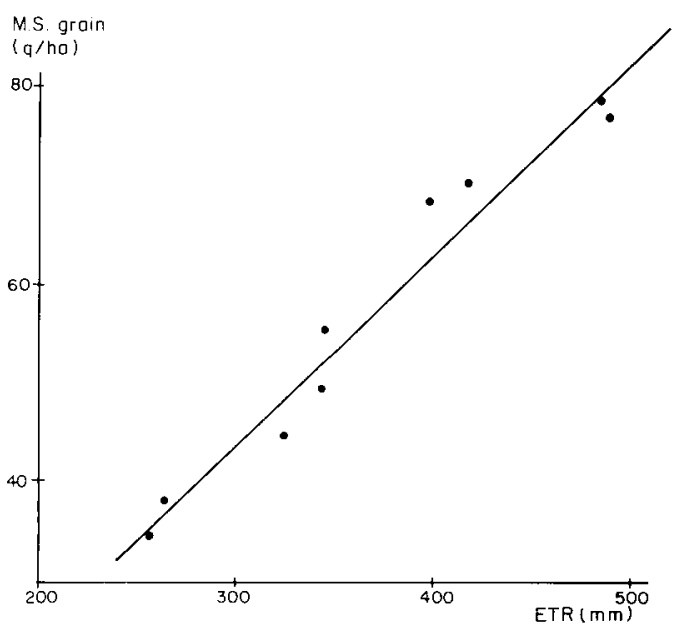

Figure 2

Relations entre le rendement du grain et l'eau consommée. Relationships between grain yield and water consumed.

\section{Relation entre rendement et alimentation azotée :} Evaluation des besoins azotés du blé d'hiver

Sur l'ensemble du dispositif, la quantité d'azote présente dans les parties aériennes est très étroitement liée au rendement du grain sẹlon la régression linéaire 
suivante : avec

$$
\mathrm{N}(\mathrm{kg} / \mathrm{ha})=3,41 \text { M.S. grain }(\mathrm{q} / \mathrm{ha})-23,2
$$

$$
\mathrm{r}=0,97 \text { et } \mathrm{n}=15 \text {. }
$$

On en déduit que chaque quintal supplémentaire de grain accroît de $3,41 \mathrm{~kg}$ l'exportation d'azote par les parties aériennes. Cependant, l'importance du terme libre dans la régression précédente engage à approfondir cette première conclusion globale.

L'analyse de la variation de la teneur du grain en azote en fonction du rendement classe les traitements du dispositif en 2 ensembles distincts selon qu'ils ont reçu $(+N)$ ou non $(-N)$ la fumure minérale de $230 \mathrm{~kg} / \mathrm{ha}$ (fig. 3). Dans le premier ensemble, la teneur varie de 2,2 à 2,7 p. 100 alors qu'elle reste comprise entre 1,5 et 1,8 dans le deuxième.

Dès lors, établie séparément sur chacun de ces ensembles, la régression linéaire précédente entre l'azote retrouvé dans les parties aériennes et le rendement du grain voit diminuer simultanément la pente et le terme libre. On obtient ainsi (fig. 4) :

- en absence de $N$ minéral $(-\mathrm{N})$

$\mathrm{N}$ exporté $(\mathrm{kg} / \mathrm{ha})=1,86 \mathrm{M} . \mathrm{S}$. grain $(\mathrm{q} / \mathrm{ha})+4,83$ avec $\mathrm{n}=4$ et $\mathrm{r}=0,98$;

- en présence de $N$ minéral $(-\mathrm{N})$

$\mathrm{N}$ exporté $=2,91$ M.S. grain $(\mathrm{q} / \mathrm{ha})+11,55$

avec $\mathrm{n}=11$ et $\mathrm{r}=0,97$.

L'examen approfondi de la teneur en azote du grain révèle la présence d'une variation secondaire au sein de l'ensemble $(+N)$ due à l'alimentation hydrique (fig. 3). Les concentrations les plus élevées, proches de 2,7 p. 100 de la M.S. sont observées sur les sols les plus touchés par la sécheresse (épaisseur de $40 \mathrm{~cm}$ ) qui, intervenant surtout après' l'anthèse, pénalise l'accumulation des hydrates de carbone. A l'inverse, en absence de sécheresse ( 80 irrigué et $160 \mathrm{~cm}$ ), les teneurs sont les plus basses, comprises entre 2,2 et 2,3 p. 100 .

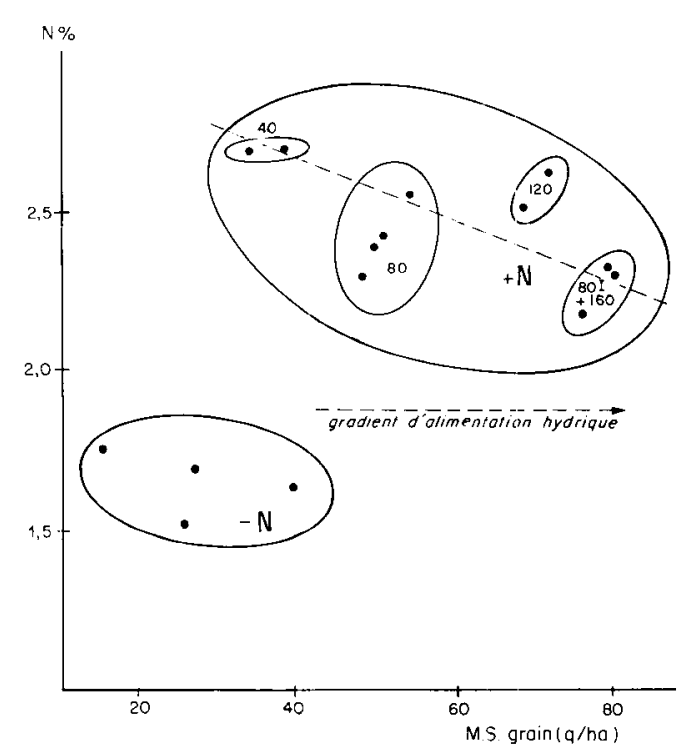

Figure 3

Relations entre la teneur en azote et le rendernent du grain.

Relationships between nitrogen content and grain yield.

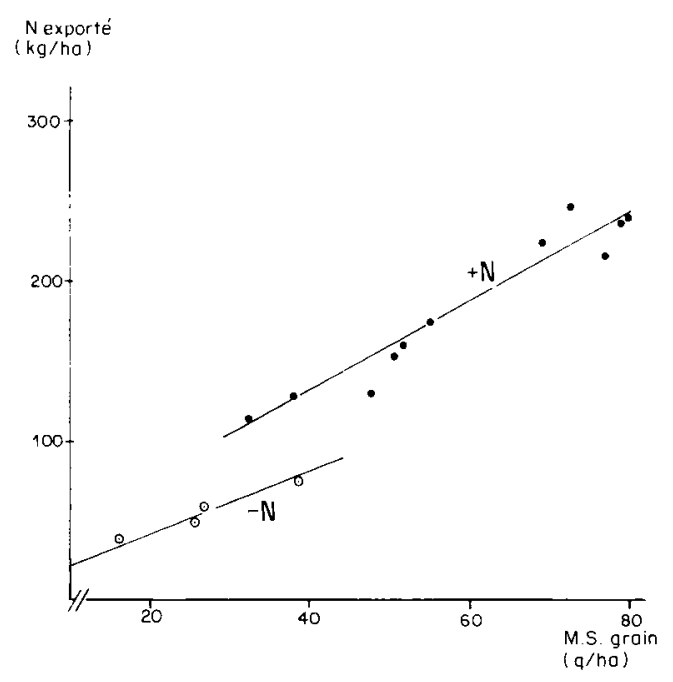

Figure 4

Relations entre l'azote exporté et le rendement du grain. Relationships between nitrogen uptake and grain yield.

En raison du trop petit nombre de traitements faisant varier les facteurs azote et eau, il n'est guère possible d'aller plus loin dans l'analyse de leur interaction. On peut néanmoins conclure qu'une teneur en azote du grain de l'ordre de 2,2 à 2,3 p. 100 paraît nécessaire pour exprimer le potentiel génétique d'une variété fourragère de blé telle que "Clément".

De plus, dans tous les traitements de l'ensemble $(+\mathrm{N})$, on constate (cf. tabl. 2) que la teneur des pailles est restée stable autour de 0,5 p. 100 correspondant à une exportation moyenne de 0,6 à $0,7 \mathrm{~kg}$ de $\mathrm{N} / \mathrm{q}$ grain produit.

L'exportation d'azote par les parties aériennes du blé ressort donc à $2,8-3,0 \mathrm{~kg} / \mathrm{q}$ de grain sec, soit une moyenne de $2,45 \mathrm{~kg} / \mathrm{q}$ grain à $15 \mathrm{p} .100$ d'humidité.

Enfin, pour évaluer les besoins totaux, il convient d'ajouter l'azote présent dans le système souterrain estimé selon CoIc (1956) à $0,6 \mathrm{~kg} / \mathrm{q}$ de grain récolté. On obtient ainsi la demande globale de $3,05 \mathrm{~kg} / \mathrm{q}$ grain à $15 \mathrm{p} .100$ d'eau, qui confirme la valeur généralement admise à la suite des travaux de cet auteur.

Ce résultat confirme aussi la signification physiologique d'une telle référence acquise puis vérifiée jusqu'à cette date exclusivement dans les conditions climatiques de plaine.

\section{CONCLUSION}

Sous réserve d'études complémentaires visant à préciser les modalités de l'élaboration du rendement en altitude, les potentialités de production du blé d'hiver - des céréales en général - apparaissent très élevées sur les plateaux du Massif Central français, proches de $100 \mathrm{q} /$ ha grain (à 15 p. 100 d'eau).

Bien entendu, ces valeurs potentielles établies sur de très petites surfaces expérimentales, soigneusement entretenues, ne sont pas transposables dans la pratique agricole sans une réfaction notable.

Le premier enseignement fourni par cette étude est l'ampleur du déficit hydrique observé en 1982, année caractérisée, il est vrai, par une fin de printemps 
moins pluvieuse que la normale (soit $259 \mathrm{~mm}$ contre $357,5 \mathrm{~mm}$ d'avril à août inclus). Il est important de souligner que ce déficit a été mesuré sur un sol relativement profond $(80 \mathrm{~cm})$ et doté d'une capacité de rétention représentative des conditions moyennes sur les plateaux du socle primaire. Une pluviosité printanière et estivale «normale » associée à un sol un peu plus profond, de l'ordre de $100 \mathrm{~cm}$, fourniraient les conditions nécessaires pour couvrir les besoins hydriques et exprimer ainsi les potentialités du blé d'hiver dans les zones d'altitude du centre de la France. Mais le blé est susceptible d'exploiter des réserves situées plus profondément encore.

D'autre part, l'azote exerce dans ces régions un rôle d'autant plus déterminant que le régime des pluies est partout favorable au lessivage de l'azote minéral du sol provenant soit des engrais soit de la minéralisation des l'humus ou des résidus culturaux. Ces conditions soulèvent notamment le problème de la valorisation optimale de l'azote apporté sous forme d'engrais de ferme et de l'azote libéré après le retournement des prairies temporaires.

Enfin, la réalisation des potentialités des cultivars modernes paraît nécessiter la fourniture minérale de $3,5 \mathrm{~kg}$ d'azote/q grain $\mathrm{sec}$ (soit $3,0 \mathrm{~kg}$ de $\mathrm{N} / \mathrm{q}$ à 15 p. 100 d'humidité), conduisant ainsi à une demande globale de l'ordre de $300 \mathrm{~kg}$ de $\mathrm{N}$ à l'hectare. Elle exige aussi une efficace protection contre les parasites et les mauvaises herbes, ces dernières ayant une propension à proliférer avec le retour des engrais de ferme sur les terres labourées des exploitations orientées vers des systèmes mixtes d'agricultureélevage.

Reçu le 5 mars 1984. Accepté le 26 septembre 1984.

\section{RÉFÉRENCES BIBLIOGRAPHIQUES}

Coic Y., 1956. La nutrition et la fertilisation azotées du blé d'hiver. 1. Les besoins en azote du blé d'hiver. Conséquences agronomiques. Ann. Agron., 1, 115-131.

Lafarge M., 1983a. Existence et diversité de la céréaliculture de montagne dans le Massif Central. C. R. Acad. Agric. Fr., 69, 500510.
Lafarge M., 1983b. Approche des facteurs et conditions du rendement des céréales en altitude. C. R. Acad. Agric. Fr., 69, 917-927.

Jacquard P., Croisier L., 1970. Etude des effets résiduels des cultures fourragères sur les cultures arables. III. Bilan de six années d'essais sur l'étude globale des effets résiduels et de leur durée. Ann. Agron., 21, 247-268. 